\title{
Singular value decomposition filtering in high-frame-rate cardiac vector flow imaging
}

\author{
Michiya Mozumi, Ryo Nagaoka, Hideyuki Hasegawa \\ Graduate School of Science and Engineering for Research, University of Toyama, Japan
}

\begin{tabular}{|c|c|}
\hline Article Info & ABSTRACT \\
\hline Article history: & Dysfunction of the left ventricle (LV) weakens the cardiac function and \\
\hline Received Aug 20, 2019 & $\begin{array}{l}\text { affects the physical activity. Echocardiagraphy has been used to visualize } \\
\text { the blood flow dynamics and to evaluate the cardiac function. }\end{array}$ \\
\hline Revised Oct 25, 2019 & However, the signal processing to suppress the clutter signals should be \\
\hline Accepted Dec 2, 2019 & $\begin{array}{l}\text { employed. In this study, we employed the singular value decomposition } \\
\text { (SVD) clutter filtering to obtain the cardiac blood speckle images. We also }\end{array}$ \\
\hline Keywords: & $\begin{array}{l}\text { employed the adaptive thresholding metric to determine the proper cutoff } \\
\text { values at each phase during the cardiac cycle. Moreover, we employed }\end{array}$ \\
\hline Block matching method & $\begin{array}{l}\text { a depth-dependent SVD clutter filter for more accurate estimation of } \\
\text { the cardiac blood echo signals. The } 2 \mathrm{D} \text { blood flow velocity vectors were }\end{array}$ \\
\hline Cardiac blood flow & estimated by applying the block matching method to obtained blood speckle \\
\hline Clutter filter & images. The obtained results show that the proposed filter suppressed \\
\hline Singular value decomposition & the clutter signals from left ventricular wall significantly, \\
\hline Ultra sound imaging & $\begin{array}{l}\text { and the contrast-to-noise ratio (CNR) was improved from }-0.5 \mathrm{~dB} \text { to } 13.8 \mathrm{~dB} \\
\text { by the proposed SVD clutter filtering. }\end{array}$ \\
\hline
\end{tabular}

This is an open access article under the CC BY-SA license.

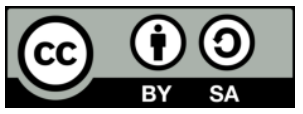

\section{Corresponding Author:}

Hideyuki Hasega wa,

Graduate School of Science and Engineering for Research,

University of Toyama,

3190 Gofuku, Toyama 930-8555, Japan.

Email: hasegawa@eng.u-toyama.ac.jp

\section{INTRODUCTION}

Echocardiography has been used to evaluate the cardiac function. The color-Doppler imaging is a credible technique for visualization of blood flow [1] and used for visualizing of the blood flowing in and out from the cardiac cavity. In echocardiography, the color-Doppler imaging is also employed to visualize an abnormal blood flow such as mitral regurgitation [2]. In the color-Doppler imaging, the flow velocity component only in the axial direction is estimated, and the velocity component parallel to the axial direction cannot be obtained. In this sense, the color-Doppler image doesn't stand for the true velocities.

The block matching method is able to estimate $2 \mathrm{D}$ velocities [3-6]. In this method, the motion of speckle patterns between successive frames is tracked and, thus, blood speckle images are required to obtain flow velocities. However, the intensity of the clutter echo is 40-60 dB higher than those originating from red blood cells [7]. Therefore, the signal processing to suppress the clutter signals should be employed. In our previous study, the high pass filter, which chooses the cut-off frequency adaptively based on the velocity of the heart wall [8], could visualize the blood B-mode images at a high-frame rate $[9,10]$.

Recently, the singular value decomposition (SVD) gains attention in research fields of image processing as a low-lank approximation method. The SVD-based filtering was applied to diagnostic imaging modalities [11, 12], and the SVD-based filter contributes to reduction of noise components contained in the diagnostic images. In the field of the medical ultrasound, the SVD-based method was employed as 
a clutter filter [13-16] and its performance is better than that of the conventional finite impulse response (FIR) filter [13, 17]. The application of the SVD clutter filter is currently limited to blood flow in small vessels [13-15]. To assess the feasibility of the SVD filter in cardiac blood flow imaging, in this study, we applied the SVD clutter filter to echo signals the received from the human heart. In clutter filtering of ultra sound echoes from the heart wall, the heart wall exhibits remarkable movements during a cardiac cycle. For this reason, the fixed cutoff values cannot be adopted in the cardiac blood flow, and the cutoff values in a clutter filter should be altered during the cardiac cycle adaptively based on the information of the clutter motion as described in our previous study [8]. To solve such a problem, we employed the adaptive thresholding metrics in the SVD clutter filter.

In the cardiac blood flow imaging, the optimum cutoff values were different at the positions of the echo data. Therefore, it is desirable to apply the clutter filters to spatially segmented ultra sound data. In this study, we employed a depth-dependent SVD clutter filter to obtain the cardiac blood echo signals. Also, an envelope-based block matching method was applied to the cardiac blood speckle images to obtain the $2 \mathrm{D}$ velocity vector maps. The performance of the proposed method was evaluated in the in vivo experiment on the human heart.

\section{RESEARCH METHOD}

\subsection{Experimental setup}

A phased array probe at a center frequency of $3 \mathrm{MHz}$ was used in the in vivo experiment. One diverging beam was transmitted using the transducer elements of the phased array probe [18, 19]. Element echo signals were received and applied the delay-and-sum (DAS) beamforming. The receiving focal points were aligned in the Cartesian coordinate to avoid a redundant effect due to non-constant lateral sampling interval in the polar coordinate [19]. As a result, beamformed RF signals along 241 scan lines were obtained. Under such conditions, a frame rate at $6250 \mathrm{~Hz}$ was achieved.

\subsection{SVD clutter filtering method}

A Let us define the beamformed RF signals $s(x, z, t)$, where $x, z$, and $t$ represent the lateral position, depth position, and time, respectively. The two-dimensional matrix $\mathbf{S}$ is created by rearranging the beamformed RF data $s(x, z, t)$ to the Casorati matrix with a size $\left(n_{x} \times n_{z}, n_{t}\right)$, where $n_{x}, n_{z}$, and $n_{t}$ is the number of pixels in the lateral and depth direction, and packet size of filter, respectively. By applying SVD, the matrix $\mathbf{S}$ can be decomposed into a production of three matrices as:

$$
S=U \Sigma V^{T}
$$

where $\mathbf{U}$ and $\mathbf{V}$ are matrices composed of spatial and temporal singular vectors. $\boldsymbol{\Sigma}$ is a diagonal matrix composed of the singular values arranged in a descending order. The superscript.$^{\mathrm{T}}$ represents transpose. The singular values obtained by such a procedure corresponds to energy of the received ultrasound signals, and the low-order, intermediate-order, and high-order singular components are assumed to be related to the clutter, blood, and noise signals, respectively. The clutter filter was accomplished by setting singula $r$ values corresponding to clutter signals to zero as:

$$
S^{\prime}=U \Sigma^{\prime} V^{T}
$$

where $\boldsymbol{\Sigma}^{\prime}$ is a diagonal matrix obtained by substituting singular values corresponding to clutter signal with zero. The filtered signals were obtained by reshaping $\mathbf{S}^{\prime}$ to the original size. Figure 1 shows a schematic of the SVD clutter filtering.

\subsection{Thresholding of SVD clutter filter}

In this section, the thresholding metric for creating the matrix $\boldsymbol{\Sigma}^{\prime}$ was introduced [15, 20]. In the SVD clutter filtering, the low-order components, which are assumed to be related to the clutter signals, were removed. Therefore, the boundaries between the clutter and blood signal components should be determined. As described in Section 1, in cardiac blood flow imaging, a cutoff threshold value of the clutter filter should be adaptively because the velocity of the heart wall changes rapidly during the cardiac cycle. 


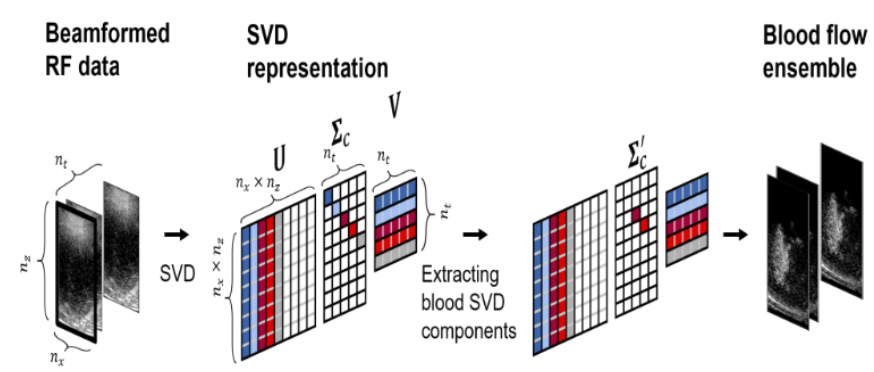

Figure 1. Schematic of the SVD clutter filtering

In this study, the blood subspace boundaries were determined based on the similarity of the spatial matrix $\mathbf{U}$. The similarity between $n$-th and $m$-th column vectors of $\mathbf{U}$ was calculated as:

$$
C(n, m)=\frac{1}{n_{x} n_{z}} \sum_{x, z}\left(\left|U_{n}(x, z)\right|-\overline{\left|U_{n}(x, z)\right|}\right) \times\left(\left|U_{m}(x, z)\right|-\overline{\left|U_{m}(x, z)\right|}\right)
$$

where $\mathbf{U}_{n}(x, z)$ is the $n$-th spatial singular vector, and - stands for a veraging. The spatial singular vector corresponds to B-mode images. The two spatial singular bases, which belong to the same subspace, have high spatial correlation. Therefore, we determined the clutter and blood signal subspaces using the spatial similarity matrix $\mathbf{C}$. Let us define the parameter matrix $\boldsymbol{\alpha}_{a, b}$ expressed as [15, 20].

$$
\boldsymbol{\alpha}_{a, b}=\left\{\begin{array}{c}
1 \text { if }(n, m) \in[1, a)^{2} \\
1 \text { if }(n, m) \in[a, b]^{2} \\
0 \text { otherwise }
\end{array}\right.
$$

The clutter and tissue and noise subspace boundaries, $a$ and $b$, were searched so that the following normalized correlation coefficient $\chi_{\mathrm{N}}$ was maximized:

$$
\chi_{N}=\frac{\chi\left(C, \boldsymbol{\alpha}_{a, b}\right)}{\sqrt{\chi^{(c, C) \cdot \chi\left(\boldsymbol{\alpha}_{a, b}, \boldsymbol{\alpha}_{a, b}\right)}}}
$$

where

$$
\chi\left(\boldsymbol{C}, \boldsymbol{\alpha}_{a, b}\right)=\frac{1}{n_{x} n_{z}} \sum_{x, z}(|\boldsymbol{C}(x, z)|-\overline{|\boldsymbol{C}(x, z)|}) \times\left(\left|\boldsymbol{\alpha}_{a, b}(x, z)\right|-\overline{\left|\boldsymbol{\alpha}_{a, b}(x, z)\right|}\right)
$$

Figure 2 shows a schematic of an adaptive thresholding metric in this study. In the clutter subspace and in the blood subspace, the spatial bases in the same subspace were highly correlated. In Figure 2, an area depicted as the red square was assigned to be the blood subspace. Then, the diagonal matrix $\boldsymbol{\Sigma}^{\prime}$ was created so that the singular values except for the blood signal components were set at zero.
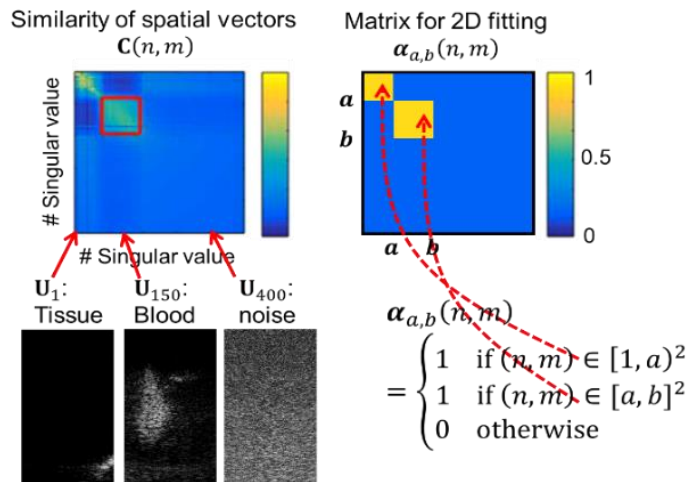

Figure 2. Schematic of the thresholding of SVD clutter filter in the case of a packet size of the filter of 500, variable a and b are chosen so that the similarity between $\boldsymbol{C}$ and $\boldsymbol{\alpha}_{\boldsymbol{a}, \boldsymbol{b}}$ is maximized 


\subsection{Depth-dependent SVD clutter filter}

In Section 2.2, the SVD clutter filter is applied to the whole region of the received ultrasound data. However, in the cardiac blood flow imaging, the optimum cutoff values were different at the spatial position $\mathrm{s}$ of the echo data [8]. Therefore, it is desirable to apply the clutter filters to spatially segmented ultrasound data. In this study, we employed a depth-dependent SVD clutter filter to obtain the cardiac blood echo signals. As shown in Figure 3(b), regions of interest (ROI) were assigned by scanning a template window in the depth direction. The ultrasound data $\mathbf{M}_{i}$ in the segmented regions were subjected to the SVD clutter filter and decomposed into a product of three matrix as:

$$
\mathrm{M}_{i}=\mathrm{U}_{i} \cdot \Sigma_{i} \cdot \mathrm{V}_{i}^{\mathrm{T}}
$$

where $i=1,2 \ldots, N_{\mathrm{ROI}}$, and $N_{\mathrm{ROI}}$ is the number of the ROIs. Blood signal components were extracted in each segmented data as:

$$
\mathrm{M}_{i}^{\prime}=\mathrm{U}_{i} \cdot \Sigma_{i}^{\prime} \cdot \mathrm{V}_{i}^{\mathrm{T}}
$$

where $\mathbf{M}_{i}^{\prime}$ is $i$-th the output signals of the SVD clutter filter. In this method, the blood signal components were estimated by the distribution of the singular values. To suppress the clutter signal components, we assigned the components with singular values of $-55 \mathrm{~dB}$ lower than the maximum singular value as the blood signal components. Also, to suppress the noise components, the noise components were estimated using the slope of the distribution of the singular values [21].

(a)
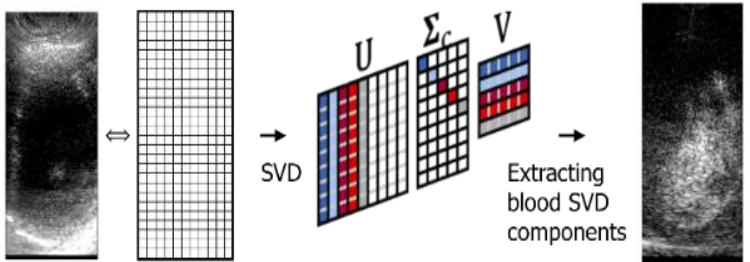

(b)

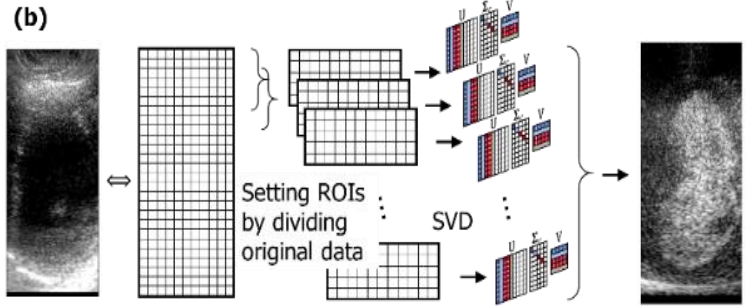

Figure 3. Schematic of the SVD filter used in this study

Finally, we concatenated output signals from each SVD filter to form one B-mode image. In this study, pixels in two adjacent ROIs were overlapped to reduce spatial discontinuity of the final output data. The numbers of overlapped pixels of adjacent ROIs were set to be $95 \%$ of a block size in the depth direction of ROI. In this case, one B-mode image was formed by concatenating $5 \%$ of output signals from each SVD filter.

\subsection{Estimation of the blood velocity vectors}

In this study, 2D velocity vectors were estimated by applying the block matching algorithm to the blood speckle images (envelope signals) obtained with the clutter filtering. The normalized correlation function between successive blood speckle frames was calculated with the kernel size of $(6.0 \mathrm{~mm} \times 7.9 \mathrm{~mm})$ in the lateral and depth directions. The obtained normalized correlations were averaged temporally to suppress undesired components and interpolated with the reconstructive interpolation to obtain the subsample displacements [22-25]. Blood velocity vectors were obtained from the interpolated correlation function ma $\mathrm{ps}$ and displayed on the blood speckle B-mode images. 


\subsection{In vivo experiment}

The ultrasound echo signals from the left ventricular of a 27 -year-old healthy volunteer were analyzed. The blood flow B-mode image were obtained by applying the SVD clutter filter to the beamformed RF signals. The B-mode image obtained with the SVD clutter filter was compared to that obtained with the FIR-based clutter filter, which chooses the cutoff frequency based on the velocity in the heart wall [8]. To evaluate the performance of the clutter filter, we measured the contrast-to-noise ratio (CNR) of the filtered images. CNR was expressed as:

$$
N R=\frac{P W\left(s_{\text {blood }}\right)-P W\left(s_{\text {tissue }}\right)}{\text { std }\left(s_{\text {tissue }}\right)}
$$

where $s_{\text {blood }}$ and $s_{\text {tissue }}$ are echo signals which correspond to blood and clutter (background), and PW $(s)$ denotes the power of the signal expressed as:

$$
P W(s)=\frac{1}{n_{x} n_{z}} \sum_{x, z}|s(x, z)|^{2}
$$

Also, $\operatorname{std}(\cdot)$ represents the standard deviation.

\section{RESULTS}

Figure 4(a)-(c) show cardiac blood flow images at a systolic phase obtained without clutter filtering (original beamformed signals), with the FIR-based filtering, and with the SVD clutter filtering, respectively. In Figure 4(c), the undesirable clutter signals at a depth of $100 \mathrm{~mm}$ were more suppressed than those shown in Figure 4(b), whereas an appearance of the blood speckles were preserved. Regions of interest (ROIs) of the blood and clutter (background) were depicted in Figure 4(a) as red rectangles. The CNRs of B-mode images in Figure 4(b) and 4(c) became -5.8 and $12.3 \mathrm{~dB}$.

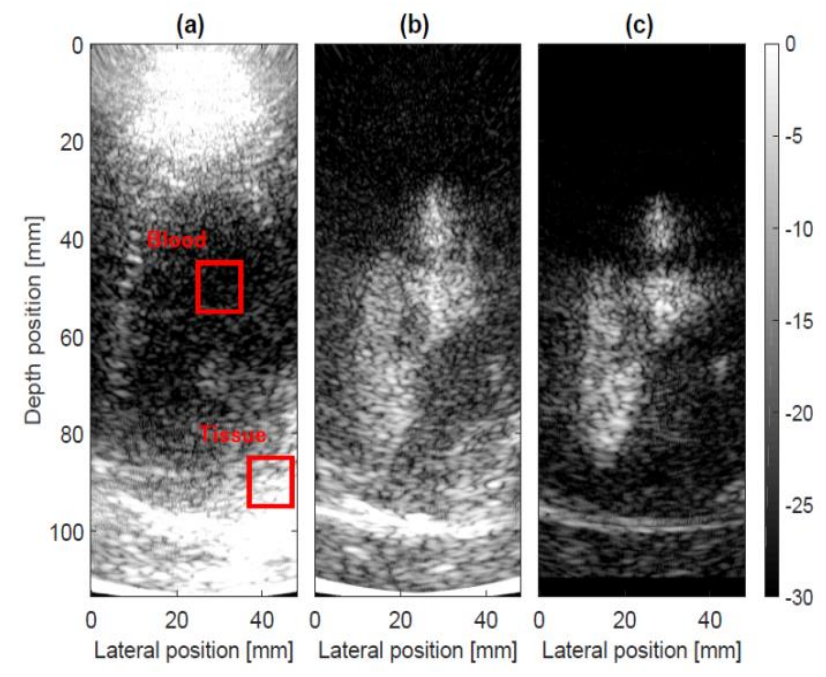

Figure 4. B-mode image of left ventricular at a systole phase, (a) Original beamformed signals,

(b) Cardiac blood flow images obtained with FIR-based filter, (c) With the SVD clutter filter, ROIs labeled with blood and tissue represent pixels corresponding to cardiac lumen and heart wall, respectively

Figure 5(a)-(c) show cardiac blood flow images at a mid-diastolic phase obtained without clutter filtering, with the FIR-based filtering, and with the SVD clutter filtering, respectively. In Figure 5(c), signals which corresponding to the heart wall were also suppressed as compared with the B-mode image in Figure 4(b). In this case, the blood speckle was less affected, but the SVD clutter filter was capable to suppress the clutter signals. The CNRs of B-mode images in Figure 5(b) and 5(c) became -0.5 and $13.8 \mathrm{~dB}$. Figure 6(b) and 6(c) show cardiac blood flow images in systolic phase obtained by the SVD clutter filter without and with the segmentation of ROI in depth direction. 


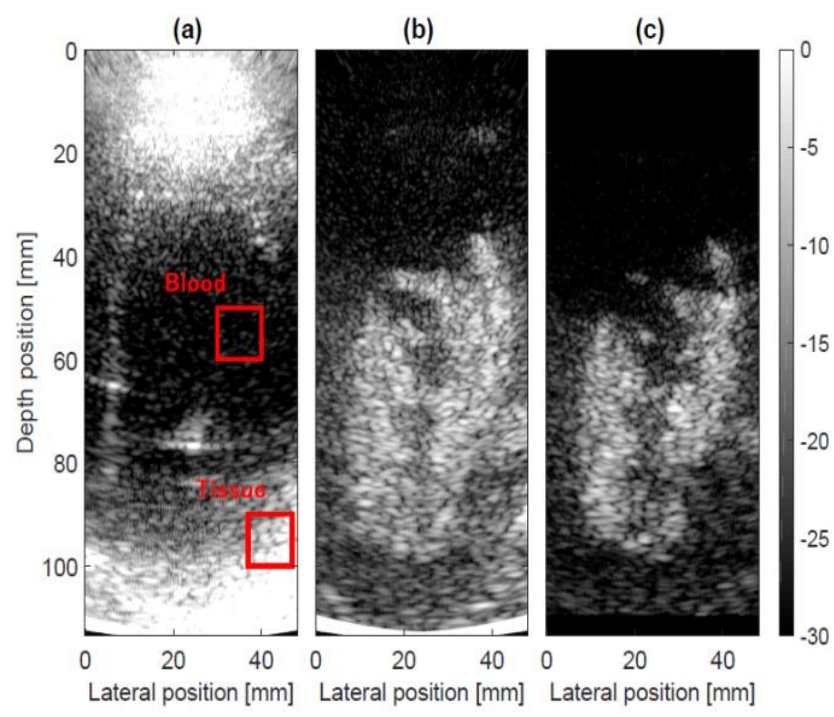

Figure 5. B-mode image of left ventricular at a mid-diastole phase, (a) Original beamformed signals,

(b) Cardiac blood flows image obtained with FIR-based filter, (c) With the SVD clutter filter

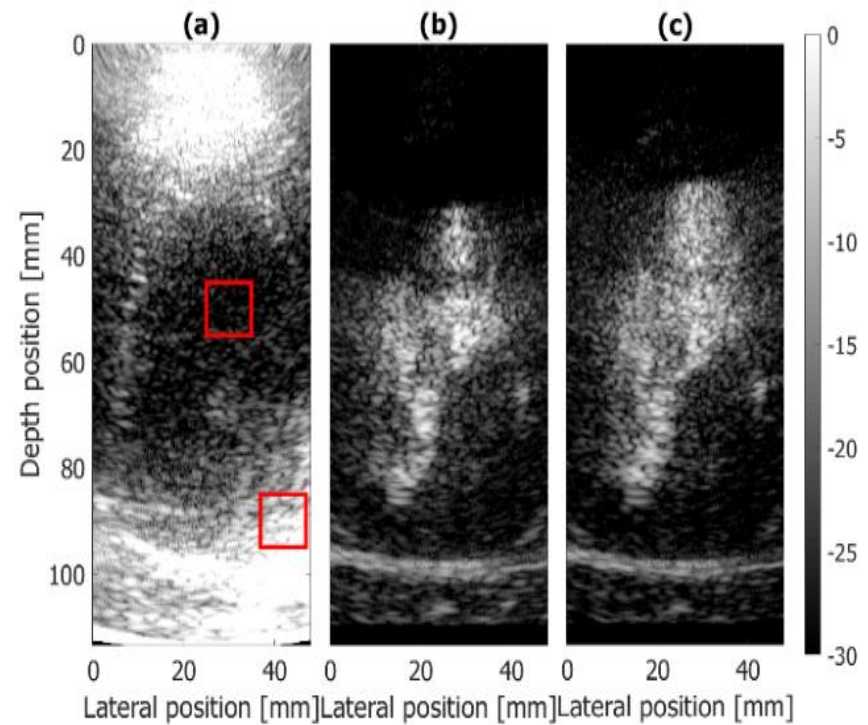

Figure 6. B-mode image of left ventricule in systole phase, (a) Original beamformed signals and obtained by SVD-based filter, (b) Without, (c) with the segmentation of ROI in depth direction

Figure 7(b) and 7(c) show cardiac blood flow images in diastolic phase obtained by the SVD clutter filter without and with the segmentation of ROI in depth direction. In those results, $n_{t}$ is set to be 512 . As shown in Figure 7(c), the contrast of echoes from blood cells is improved than Figure 7(b) by applying the depth-dependent SVD clutter filters to each ROIs. The CNRs of B-mode images in Figure 6(b) and 6(c) became 17.2 and $20.0 \mathrm{~dB}$, and Figure 7(b) and 7(c) became 9.7 and $14.9 \mathrm{~dB}$. Figure 8(a) and 8(b) show the 2D blood velocity vectors of the cardiac blood flow at a mid-diastolic phase obtained with the FIR-based filtering and with SVD clutter filtering. In Figure 8(b), vortex flows in the left ventricle were captured well in the proposed method. 


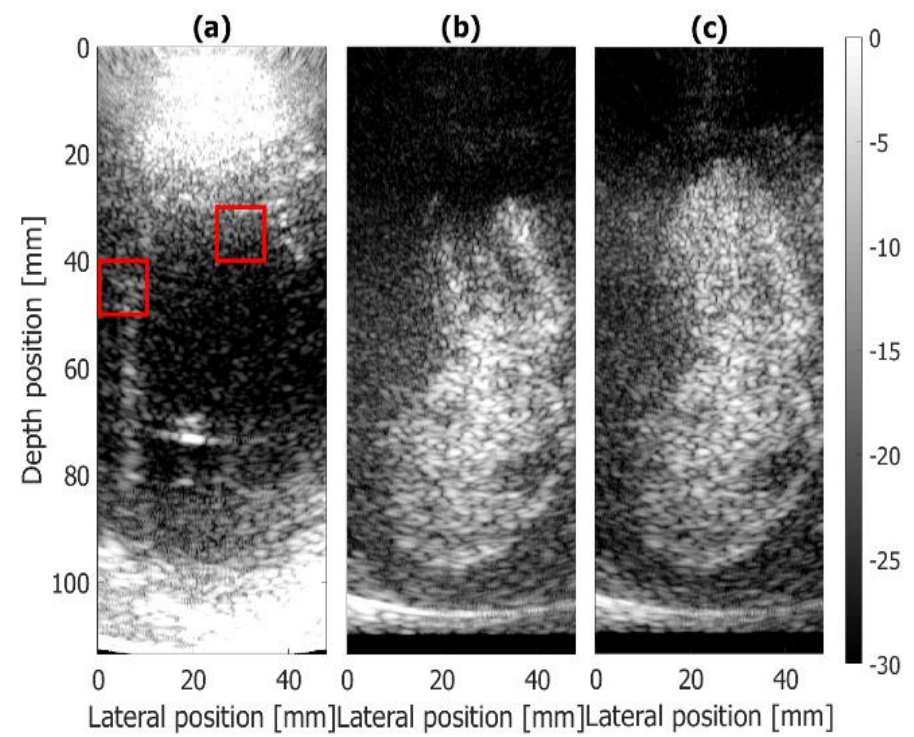

Figure 7. B-mode image of left ventricule in early-diastole phase, (a) Original beamformed signals and obtained by SVD-based filter, (b) Without, (c) With the segmentation of ROI in depth direction

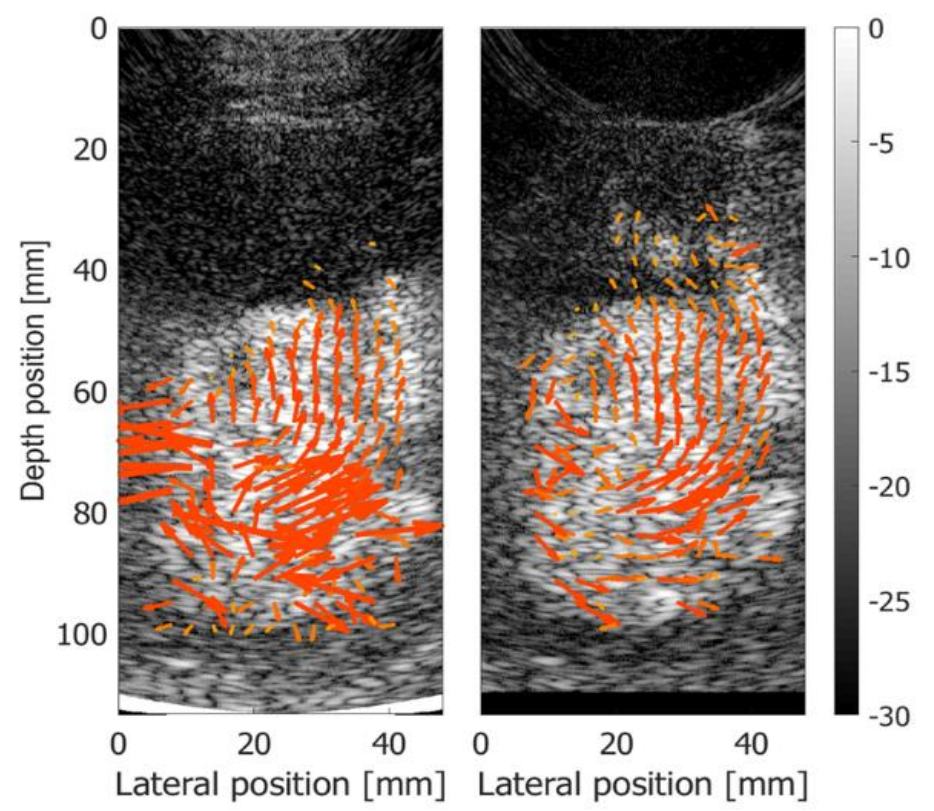

Figure 8. 2D blood velocity vectors on the blood speckle image obtained with, (a) FIR-based filter and with,

(b) The SVD clutter filtering. Velocity vectors were obtained with the block matching technique

\section{CONCLUSION}

Visualization of the blood flow vector is useful to evaluate behavior of the cardiac blood flow. In this study, we applied the SVD-based clutter filter to the ultra sound echo signals from the human heart to obtain the cardiac blood flow image. We also employed the adaptive thresholding metric to determine the proper cutoff values through the cardiac cycle. Moreover, we employed a depth-dependent SVD clutter filter for more accurate estimation of the cardiac blood echo signals. The experimental results show that the proposed filter suppressed the clutter signals from left ventricular wall, and the CNR evalua ted using the blood speckle image was improved from $-0.5 \mathrm{~dB}$ to $13.8 \mathrm{~dB}$ by the SVD clutter filtering. 


\section{ACKNOWLEDGEMENTS}

This study was supported by JSPS KAKENHI Grant Number JP17H03276.

\section{REFERENCES}

[1] D. H. Evans, J. A. Jensen, and M. B. Nielsen, "Ultrasonic Colour Doppler Imaging," Interface Focus, vol. 1, no. 4, pp. 490-502, 2011.

[2] R. Omoto, Y. Yokote, S. Takamoto, S. Kyo, K. Ueda, H. Asano, K. Namekawa, C. Kasai, Y. Kondo, and A. Koyano, "The Development of Real-Time Two-Dimensional Doppler Echocardiography and its Clinical Significance in Acquired Valvular Diseases with Special Reference to the Evaluation of Valvular Regurgitation," Japann Heart Journal, vol. 25, no. 3, pp. 325-340, 1984.

[3] L. N. Bohs, B. J. Geiman, M. E. Anderson, S. C. Gebhart, and G. E. Trahey, "Speckle Tracking for MultiDimensional flow Estimation," Ultrasonics, vol. 38, no. 1-8, pp. 369-375, 2000.

[4] S. Fadnes, M. S. Wigen, S. A. Nyrnes and L. Lovstakken, "In Vivo Intracardiac Vector Flow Imaging Using Phased Array Transducers for Pediatric Cardiology," in IEEE Transactions on Ultrasonics, Ferroelectrics, and Frequency Control, vol. 64, no. 9, pp. 1318-1326, Sept. 2017.

[5] H. Takahashi, H. Hasegawa, and H. Kanai, "Temporal Averaging of Two-Dimensional Correlation Functions for Velocity Vector Imaging of Cardiac Blood Flow," Journal of Medical Ultrasonics, vol. 42, no. 3, pp. 323-330, 2015.

[6] J. Albinsson, H. Hasegawa, H. Takahashi, E. Boni, A. Ramalli, Å. R. Ahlgren, and M. Cinthio, "Iterative 2D Tissue Motion Tracking in Ultrafast Ultrasound Imaging," Applied Sciences, vol. 8, no. 5, pp. 1-16, 2018

[7] L. A. F. Ledoux, P. J. Brands, and A. P. G. Hoeks, "Reduction of the Clutter Component in Doppler Ultrasound Signals Based on Singular Value Decomposition: A Simulation Study," Ultrasonic Imaging, vol. 19, no. 1, pp. 1-18, 1997.

[8] H. Takahashi, H. Hasegawa, and H. Kanai "Echo Motion Imaging with Adaptive Clutter Filter for Assessment of Cardiac Blood Flow," Japanese Journal of Applied Physics, vol. 54, no. 7, pp.1-8, 2015.

[9] H. Takahashi, H. Hasegawa, and H. Kanai, "Echo Speckle Imaging of Blood Particles with High-Frame-Rate Echocardiography," Japanese Journal of Applied Physics, vol. 53, no. 7, pp. 1-7, 2014.

[10] J. A. Jensen, S. I. Nikolov, A. C. H. Yu and D. Garcia, "Ultrasound Vector Flow Imaging-Part II: Parallel Systems," in IEEE Transactions on Ultrasonics, Ferroelectrics, and Frequency Control, vol. 63, no. 11, pp. 1722-1732, Nov. 2016.

[11] R. Otazo, E. Candes, and D. K. Sodickson "Low-Rank Plus Sparse Matrix Decomposition for Accelerated Dynamic MRI with Separation of Background and Dynamic Components," Magnetic Resonance in Medicine, vol. 73, no. 3, pp. 1125-1136, 2014.

[12] H. Gao, H. Yu, S. Osher, and G. Wang "Multi-Energy CT based on a Prior Rank, Intensity and Sparsity Model (PRISM)," Inverse Problems, vol. 27, no. 11, pp. 115012, 2011

[13] C. Demené et al., "Spatiotemporal Clutter Filtering of Ultrafast Ultrasound Data Highly Increases Doppler and fUltrasound Sensitivity," in IEEE Transactions on Medical Imaging, vol. 34, no. 11, pp. 2271-2285, Nov. 2015.

[14] P. Song et al., "Accelerated Singular Value-Based Ultrasound Blood Flow Clutter Filtering With Randomized Singular Value Decomposition and Randomized Spatial Downsampling," in IEEE Transactions on Ultrasonics, Ferroelectrics, and Frequency Control, vol. 64, no. 4, pp. 706-716, April 2017.

[15] J. Baranger, B. Arnal, F. Perren, O. Baud, M. Tanter and C. Demené, "Adaptive Spatiotemporal SVD Clutter Filtering for Ultrafast Doppler Imaging Using Similarity of Spatial Singular Vectors," in IEEE Transactions on Medical Imaging, vol. 37, no. 7, pp. 1574-1586, July 2018.

[16] H. Ikeda, R. Nagaoka, M. Lafond, S. Yoshizawa, R. Iwasaki, M. Maeda, S. Umemura, and Y. Saijo, "Singular Value Decomposition of Received Ultrasound Signal for Separation Among Tissue, Blood Flow and Cavitation Signals," Japanese Journal of Applied Physics, vol. 57, no. 7S1, pp. 1-6, 2018.

[17] A. C. H. Yu and L. Lovstakken, "Eigen-based clutter filter design for ultrasound color flow imaging: a review," in IEEE Transactions on Ultrasonics, Ferroelectrics, and Frequency Control, vol. 57, no. 5, pp. 1096-1111, May 2010.

[18] H. Hasegawa and H. Kanai, "High-Frame-Rate Echocardiography using Diverging Transmit Beams and Parallel Receive Beamforming," Journal of Medical Ultrasonics, vol. 38, no. 3, pp. 129-140, 2011.

[19] K. Kaburaki, M. Mozumi, and H. Hasegawa, "Estimation of Two-Dimensional Motion Velocity using Ultrasonic Signals Beamformed in Cartesian Coordinate for Measurement of Cardiac Dynamics," Japanese Journal of Applied Physics, vol. 57, no. 7S1, pp. 1-6, 2018

[20] M. Mozumi, R. Nagaoka, and H. Hasegawa, "Initial Phantom Study on Estimation of Speed of Sound in Medium using Coherence among Received Echo Signals," Japanese Journal of Applied Physics, vol. 58, pp. 1-8, 2019.

[21] R. Nagaoka and H. Hasegawa, "Identification of Vascular Lumen by Singular Value Decomposition Filtering on Blood Flow Velocity Distribution," Journal of Medical Ultrasononics, vol. 46, pp. 187-194, 2018.

[22] I. Céspedes, Y. Huang, J. Ophir, and S. Spratt, "Methods for Estimation of Subsample Time Delays of Digitized Echo Signals," Ultrason. Imaging, vol. 17, no. 2, pp. 142-171, 1995.

[23] Y. Honjo, H. Hasegawa, and H. Kanai, "Two-Dimensional Tracking of Heart Wall for Detailed Analysis of Heart Function at High Temporal and Spatial Resolutions," Japanese Journal of Applied Physics, vol. 49, no. 7, pp. 1-9, 2010. 
[24] A. Miyajo and H. Hasegawa, "Measurement of 2D Motion of Carotid Arterial Wall using Phase Shift and Frequency of Ultrasonic Echo," Japanese Journal of Applied Physics, vol. 57, pp. 1-8, 2018.

[25] A. Miyajo, R. Nagaoka, and H. Hasegawa, "Comparison of Ultrasonic Motion Estimators for Vascular Applications," Japanese Journal of Applied Physics, vol. 58, pp. 1-6, 2019.

\section{BIOGRAPHIES OF AUTHORS}

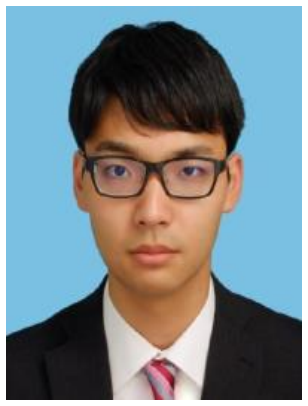

Michiya Mozumi was born in Gifu, Japan, in 1995. He received the B.S. degree in intellectual informations engineering from Toyama University, Gofuku, Japan, in 2018, He currently pursues the M.S. degree in the intellectual information engineering from Toyama University. He is a member of the Acoustical Society of Japan.

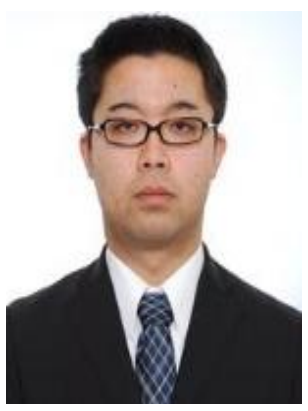

Ryo Nagaoka received the B.S. degree in electrical engineering from Tohoku University, Sendai, Japan, in 1996. He received the Ph.D. degrees in biomedical engineering from Tohoku University in 2016. From 2014 to 2016, he was a JSPS Research Fellow with the Department of Biomedical Engineering, Tohoku University. He has been an assistant professor at the Graduate School of Science and Engineering for Research, University of Toyama from 2018. His current research interests include the photoacoustic imaging, electrography, and ultrasound microscopy.

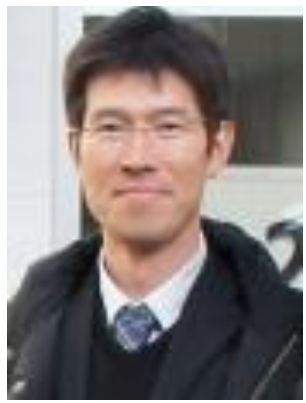

Hideyuki Hasegawa received the B.E. degree from Tohoku University, Sendai, Japan, in 1996. He received the Ph.D. degree from Tohoku University in 2001. He received the research fellowships for young scientists (DC1) and (PD) from the Japan Society for the Promotion of Science in 1998 and 2001, respectively. He became a research associate and associate professor in 2002 and 2007 at the Graduate School of Engineering, Tohoku University. From 2008, he became an associate professor at the Graduate School of Biomedical Engineering, Tohoku University. He has been a professor at Graduate School of Science and Engineering for Research, University of Toyama from 2015. 